\title{
Hardiness as a Factor of Psychological Resilience of Youth in Conditions of Military Conflict
}

\author{
Svitlana Kravchuk \\ Institute for Social and Political Psychology, NAES of Ukraine, Kyiv, Ukraine
}

Introduction. The study of the psychological resilience and hardiness of the personality is especially relevant in connection with the military conflict in the East of Ukraine, which has led to the destruction of social order, social structure of communities, and, for many, the loss of contact with close people. In youth the formation of moral self-awareness and self-regulation, which become more complete and meaningful with age, allow the individual to form his or her own worldview. During this period the social position of the individual changes, causing a change in the significance of any teaching; the process of self-determination is intensified with self-esteem, self-awareness, psychological readiness for life, and identity formation. The study of the peculiarities of hardiness and psychological resilience of youth is particularly important when we consider the negative impacts of military conflict.

Purpose. Our purpose was to understand and establish the peculiarities of hardiness as a factor of the psychological resilience of young people living in the conditions of a military conflict.

Methodology. 123 young people (from 19 to 23 years old) took part in our empirical study students of Taras Shevchenko Kyiv National University and Borys Grinchenko Kyiv University. To study psychological resilience we used the test, "Diagnostics of psychological resilience" (S. Kravchuk, O. Osadko). The Cronbach's Alpha indicates that the internal consistency of the scale of psychological resilience is acceptable (Cronbach's Alpha = 0,775). To study hardiness and its components we used the test "Diagnostics of hardiness" (S. Maddi). Significant direct correlative relations between psychological resilience and hardiness (Spearman's Correlation Coefficient $=0,718$, sig $(p)<0,001)$ and its components commitment (Spearman's Correlation Coefficient $=0,684$, sig $(p)<0,001$ ), control (Spearman's Correlation Coefficient $=0,656$, sig $(p)<0,001$ ), and challenge (Spearman's Correlation Coefficient $=0,523$, sig $(\mathrm{p})<0,001)$ are revealed. Based the use of multiple regression analysis, we established that challenge (Standardized Beta Coefficient $=0,322$, sig (p) < 0,001), commitment (Standardized Beta Coefficient $=0,295$, sig (p) <0,01) and control (Standardized Beta Coefficient $=0,270$, sig $(p)<0,01$ ) are determinants of psychological resilience of youth. In this research, it was empirically discovered that the higher the hardiness of young people as described above, the less likely they are to engage in critical provocative situations in such forms of emotional response as anger, impotence, fear, and irritation and also less prone to active aggression as a form of behavioural response. The direct significant correlative link of hardiness with satisfaction with quality of life was revealed. A person with a high level of hardiness is characterized by optimism and activity of a vital position.

Results and Discussion. Concepts of psychological resilience and hardiness are not synonymous. We consider psychological resilience as an integrative feature of the individual which manifests itself in the ability to maintain a stable level of psychological and physical 
functioning in critical situations, to come out of such situations without persistent violations, to successfully adapt to adverse changes. The concept of psychological resilience describes the ability to overcome difficulties, to adapt positively, and to function successfully in critical situations.

We believe that the concept of hardiness should be considered as a psychological phenomenon that at the content-structural level of analysis is a complex integral characteristic of an individual, which includes emotional, cognitive, moral, volitional, behavioural components. The emotional component of hardiness includes emotional stability, stress resistance, equilibrium, commitment, and an optimistic view of life. The cognitive component of hardiness includes control, challenge, persuasion, expectation, attribution, and self-relation. The volitional component of hardiness includes purposefulness, initiative, independence, resolve, perseverance, endurance, resistance. The moral component of hardiness includes humanistic values. Finally, the behavioural component includes active interaction with the surrounding reality.

At the process-dynamic level of analysis, hardiness, as a psychological phenomenon, acts as a personal style. At the functional level of analysis, hardiness as a psychological phenomenon serves as a component of adaptive personal resourcefulness, the creative potential of the individual, personal maturity and as a predictor of psychological resilience - an indicator of mental health, a functional component of viability and a factor of life creativity.

Practical value. The practical value of the study is that the results of this empirical research can be used in the process of further developing an understanding of the function of characteristics of hardiness as a factor of the psychological resilience of youth. The scientific data obtained related to the peculiarities of hardiness and psychological resilience in young people will contribute to the development of a training program aimed at developing psychological resilience in youth.

Conclusion. In future we plan to continue the study of peculiarities of hardiness and psychological resilience of individuals in the situation of military conflict.

Keywords: hardiness, personality, psychological resilience, youth, commitment, control, challenge, military conflict 